\title{
CORRIGENDUM
}

\section{On new scaling laws in a temporally evolving turbulent plane jet using Lie symmetry analysis and direct numerical simulation - CORRIGENDUM}

\author{
H. Sadeghi, M. Oberlack and M. Gauding \\ doi:10.1017/jfm.2018.625, Published by Cambridge University Press, \\ 6 September 2018
}

We recently published a paper (Sadeghi, Oberlack \& Gauding 2018) on the context of symmetry in the temporally evolving plane turbulent jets. A reader's observation through the editor brought to our attention that the use of one of the additional symmetries in second moments is nonphysical in the jet flow we studied, and a correction is necessary. While the symmetry approach and the trend to the derivation of scaling laws are not altered, we make an important theoretical correction that significantly affects a conclusion of the original paper with respect to the second moments and one of the figures presented (figure 7, p. 251). Hereby, we identify the error and present the corrected version as needed.

In the original manuscript, the following relations were derived for the second moments (4.10)-(4.12):

$$
\begin{gathered}
\tilde{R}_{11}^{0}\left(\tilde{x}_{2}\right)=-D\left(R_{11}^{0}+\bar{U}_{1}^{2}\right)\left(t-t_{0}\right)+a_{H_{11}} t, \\
\tilde{R}_{22}^{0}\left(\tilde{x}_{2}\right)=-D R_{22}^{0}\left(t-t_{0}\right)+a_{H_{22}} t \\
\tilde{R}_{33}^{0}\left(\tilde{x}_{2}\right)=-D R_{33}^{0}\left(t-t_{0}\right)+a_{H_{33}} t .
\end{gathered}
$$

It is now apparent to us that the constants $a_{H_{[i i]}}$, which are independent symmetry group parameters and were used to construct scaling laws in the original manuscript, are nonphysical in the present jet flow as they lead to large discrepancy of the profiles at large $\tilde{x}_{2}$ and $t$. As for the correction, we set the constants to $a_{H_{[i]}}=0$, which gives the corrected scaling laws

$$
\begin{gathered}
\tilde{R}_{11}^{0}\left(\tilde{x}_{2}\right)=-D\left(R_{11}^{0}+\bar{U}_{1}^{2}\right)\left(t-t_{0}\right), \\
\tilde{R}_{22}^{0}\left(\tilde{x}_{2}\right)=-D R_{22}^{0}\left(t-t_{0}\right), \\
\tilde{R}_{33}^{0}\left(\tilde{x}_{2}\right)=-D R_{33}^{0}\left(t-t_{0}\right)
\end{gathered}
$$

in place of the relations (4.10)-(4.12). Additionally, figure 7, for which we have processed the DNS data according to the right-hand side of the formulas (C.4)-(C.6), is re-plotted and shown here as figure 1. It can be observed that the self-similarity for $\tilde{R}_{11}^{0}\left(\tilde{x}_{2}\right)$ is very good but that the similarity for $\tilde{R}_{22}^{0}\left(\tilde{x}_{2}\right)$ and $\tilde{R}_{33}^{0}\left(\tilde{x}_{2}\right)$ is no better than has been derived previously. Therefore, and based on our current knowledge of 

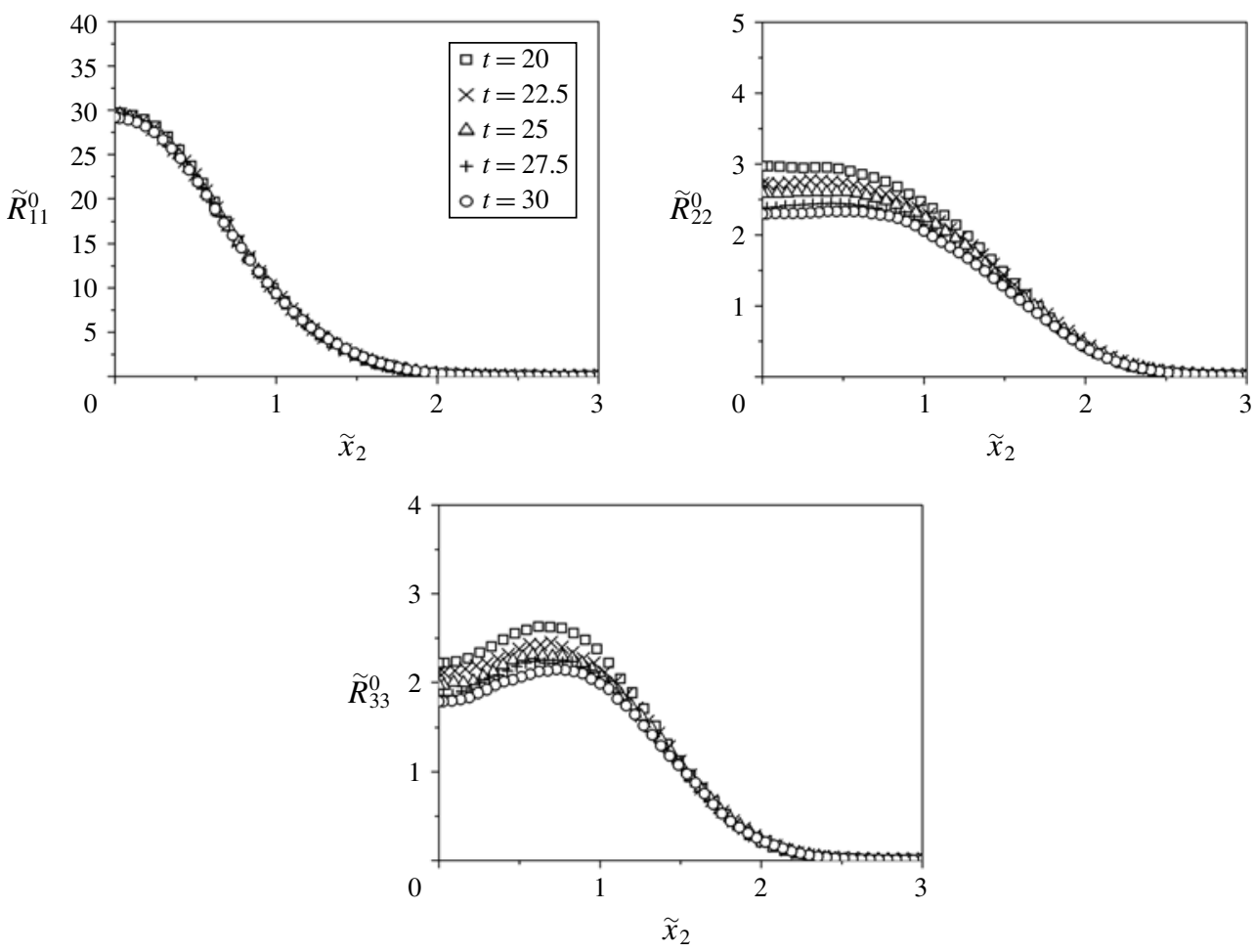

FIGURE 1. The self-similar solution (C.4)-(C.6) compared to the DNS data.

symmetries for the present flow, we emphasize the DNS data cannot exhibit a perfect collapse with the obtained scaling laws for $R_{22}^{0}$ and $R_{33}^{0}$, which is contrary to the conclusion of the original paper that 'The DNS data exhibit a perfect collapse with the obtained scaling laws'. Consequently, the formulas (4.10)-(4.12) and figure 7 of the original paper were not theoretically correct. In the context of the second moments, the main conclusion here is that current analysis only provides a very good collapse for $R_{11}^{0}$ and $R_{12}^{0}$ (as shown in the original paper in figure 6), whereas the components $R_{22}^{0}$ and $R_{33}^{0}$ are not much improved compared to the classical solutions.

\section{Acknowledgement}

Finally, the authors thank M. Frewer for the friendly hint to the error in the original paper.

\section{REFERENCE}

Sadeghi, H., Oberlack, M. \& Gauding, M. 2018 On new scaling laws in a temporally evolving turbulent plane jet using Lie symmetry analysis and direct numerical simulation. J. Fluid Mech. 854, 233-260. 